\title{
Childhood CCL18, CXCL10 and CXCL11 levels differentially relate to and predict allergy development
}

\author{
Johanna Huoman $^{1} \odot$ | Sadia Haider ${ }^{2}$ | Angela Simpson ${ }^{3}$ | Clare S. Murray ${ }^{3}$ | \\ Adnan Custovic $^{2}$ (1) | Maria C. Jenmalm ${ }^{1}$ () \\ ${ }^{1}$ Division of Inflammation and Infection, Department of Biomedical and Clinical Sciences, Linköping University, Linköping, Sweden \\ ${ }^{2}$ National Heart and Lung Institute, Imperial College London, London, UK \\ ${ }^{3}$ Division of Infection, Immunity and Respiratory Medicine, Faculty of Biology, Medicine and Health, Manchester Academic Health Sciences Centre, University \\ of Manchester and University Hospital of South Manchester NHS Foundation Trust, Manchester, UK
}

\begin{abstract}
Correspondence
Johanna Huoman, Division of Inflammation and Infection, Department of Biomedical and Clinical Sciences, Faculty of Medicine and Health Sciences, Linköping University, Lab 1, Building 420, entrance 68, level 12, 58183 Linköping, Sweden.

Email: johanna.huoman@liu.se

Funding information

MAAS is supported by MRC grants MR/ L012693/1, MR/K002449/2 and MR/ S025340/1 and Manchester Biomedical Research Centre (BRC). The views expressed are those of the author(s) and not necessarily those of the NIHR or the Department of Health and Social Care. The analyses in this study were supported by grants from the Swedish Research Council (2016-01698), the Swedish HeartLung Foundation (20140321), the Cancer and Allergy Foundation, the Foundation Samariten and the Ellen, Walter and Lennart Hesselman foundation. None of the above-mentioned supporting funders have neither been involved in the sample collection, planning, execution and interpretation of the data, nor in the preparation of this manuscript.
\end{abstract}

Editor: Ömer Kalaycı

\begin{abstract}
Background: Chemokines are important mediators in immune cell recruitment, contributing to allergy development. However, extensive studies of chemokines in the circulation in relation to the presence and development of allergic diseases remain scarce. Our aim was to investigate associations of circulating allergy-related chemokines with the development of asthma and sensitization cross-sectionally and longitudinally in a population-based cohort.

Methods: The chemokines CCL17, CCL22, CXCL10, CXCL11 and CCL18 were measured in plasma samples from children in the Manchester Asthma and Allergy Study. Samples were available from cord blood at birth $(n=376)$, age $1(n=195)$ and age 8 $(n=334)$. Cross-sectional and longitudinal association analyses were performed in relation to asthma and allergic sensitization, as well as allergic phenotype clusters previously derived using machine learning in the same study population.

Results: In children with asthma and/or allergic sensitization, CCL18 levels were consistently elevated at 1 and/or 8 years of ages. In a longitudinal model including information on asthma from 4 time points (5, 8, 11 and 16 years of ages), we observed a significant association between increasing CCL18 levels at age 1 and a higher risk of asthma from early school age to adolescence (OR $=2.9,95 \% \mathrm{Cl} 1.1-7.6, p=.028)$. We observed similar associations in longitudinal models for allergic sensitization. Asthma later in life was preceded by increased CXCL10 levels after birth and decreased CXCL11 levels at birth.

Conclusion: Elevated CCL18 levels throughout childhood precede the development of asthma and allergic sensitization. The Th1-associated chemokines CXCL10 and
\end{abstract}

Huoman and Haider shared first authorship. 
CXCL11 also associated with the development of both outcomes, with differential temporal effects.

\section{KEYWORDS}

allergy, asthma, CCL18, chemokine, CXCL10, CXCL11, sensitization

\section{1 | INTRODUCTION}

Worldwide, allergic diseases affect development and well-being of many children and adolescents, while also posing a considerable socio-economic burden. ${ }^{1}$ Allergic diseases are heterogeneous and may present with similar symptoms but different underlying causes, the features of which are just beginning to be characterized. ${ }^{2}$ Both sensitization and asthma are considered heterogeneous traits, with distinct clusters being unveiled using unsupervised machine learning techniques. ${ }^{3,4}$ While numerous immune biomarkers have been investigated as potential determinants of heterogeneity and development of allergic phenotypes, thus far none has proven unequivocal utility in a clinical setting.

Allergic inflammation depends on chemokine-facilitated recruitment of immune cells to the allergic reaction site. ${ }^{5}$ The development of allergic symptoms and sensitization early in life is preceded by elevated cord blood levels of the Th2-associated chemokines CCL17 and CCL22, respectively, ${ }^{6,7}$ with similar findings from sensitized children with allergic symptoms, and children developing recurrent wheeze ${ }^{8}$ and asthma. ${ }^{7}$ Likewise, increased levels of Th1-associated chemokines CXCL10 and/or CXCL11 have been observed in both viral-induced ${ }^{9}$ and moderate-to-severe asthma. ${ }^{10}$ Multiple studies have also revealed elevated early-life Th1-associated chemokine levels predicting outcomes such as wheezing and asthma. ${ }^{8,11}$ In contrast, sensitized children revealed lower circulating levels of CXCL11 at birth and 2 years of age, ${ }^{8}$ suggesting diverse mechanisms of action of these chemokines in allergy development. CCL18 is under dual regulation of both Th2 and Treg cells, ${ }^{12}$ and at steady state, it is mainly a regulatory chemokine, which is up-regulated in allergic conditions such as allergic rhinitis, atopic dermatitis and asthma. ${ }^{12}$ Indeed, we have previously showed elevated levels of CCL18 in children developing eczema and recurrent wheeze in the first years of life. ${ }^{8}$

Although the chemokines CXCL10, CXCL11, CCL17, CCL22 and CCL18 have previously been characterized as important mediators in allergy development, thus far they have not been studied in a larger population-based cohort to establish their role in a longitudinal setting. To this end, we measured circulating levels of these chemokines at three time points throughout childhood (at birth, 1 year and 8 years of age) in a population-based birth cohort ${ }^{13}$ and related these chemokines to allergic outcomes from infancy to 16 years of age. Furthermore, we ascertained the relationship between these chemokines and previously described clusters of allergic diseases derived using machine learning in this cohort. ${ }^{14-17}$ We hypothesized that levels of the allergy-related chemokines

\section{Key Message}

Chemokines are highly involved in the development of allergic disease, by promoting recruitment to the allergic reaction site. While previous studies have shown associations of childhood chemokine levels with the development of allergic manifestations, we were in this study able to show longitudinal impact of chemokine levels in infancy and childhood on the development of sensitization and asthma both contemporaneously and later in life. This suggests chemokines as important biomarkers for allergic disease, which could potentially be useful in a clinical setting for predicting development of sensitization and asthma.

will precede the development of different allergic phenotypes throughout childhood.

\section{2 | METHODS}

Details on the materials, methods, definitions of variables and statistical approach may be found in the Supplementary Appendix. Briefly, 905 plasma samples originating from the time of birth (cord blood, $n=376), 1$ year $(n=195)$ and 8 years $(n=334)$ from the Manchester Asthma and Allergy Study (MAAS) were analysed for their chemokine content (Figure S1). Plasma levels of CCL18 were measured using an in-house DuoSet ELISA kit (R \& D Systems), and an in-house multiplex bead assay was set up for the analysis of circulating CCL17, CCL22, CXCL10 and CXCL11.

The chemokine levels were thereafter associated with asthma and allergic sensitization, and CCL18 levels were additionally related to multivariable outcomes. To study whether chemokine levels predicted asthma or allergic sensitization, binomial logistic regression analyses were performed on natural log-transformed chemokine data. Longitudinal analyses were performed using generalized estimating equations (GEE). Population-averaged GEE models were developed to investigate whether the effect of natural log-transformed chemokine levels on the development of asthma or sensitization changed over time. All models were adjusted for parental atopy, parental smoking and gender. Resulting coefficients represent the increased/decreased odds of the respective outcome per log-unit increase in chemokine levels. 
Analyses were conducted in GraphPad Prism 8, IBM SPSS Statistics version 25 and Stata 15 software.

\section{3 | RESULTS}

\section{1 | Kinetics of circulating chemokines}

The plasma measurements revealed distinctive expression patterns for the five chemokines (Figure 1). The Th2-associated chemokine CCL17 displayed significantly lower levels at 1 and 8 years of age, compared to at birth (Figure 1A). In contrast, the Th2-associated chemokine CCL22 revealed higher concentrations at age 8 compared to earlier in life (Figure 1B). For the Th2/Treg-associated chemokine CCL18 and the Th1-associated chemokine CXCL10, significantly higher levels were evident at 1 and 8 years of age compared to at birth (Figure 1C and D). However, the expression was significantly lower at age 8 than at age 1 for both chemokines. The Th1-associated chemokine CXCL11 showed significantly higher levels at age 8 compared to the earlier time points (Figure 1E).

\section{2 | CXCL10, CXCL11 and CCL18 levels associate with asthma and sensitization}

As the investigated chemokines have previously been related to allergy development, we performed cross-sectional analyses in relation to the outcomes of asthma (Figure 2 and S2) and sensitization (Figure 3 and S3) in childhood and adolescence. Overviews of significant differences of chemokine levels in relation to allergy outcomes are shown in Figures 2A and 3A for asthma and sensitization, respectively.

\subsection{1 | Asthma}

CXCL10 levels were significantly increased at 1 year of age in children with asthma at the same age (Figure S2A). Furthermore, children with asthma at 8 and 11 years of ages exhibited elevated CXCL10 levels at age 8 (Figure 2B, Figure S2B). In contrast, CXCL11 was significantly decreased in cord blood of children having asthma at age 16 (Figure S2C). For CCL18, higher levels at age 8 associated with asthma at 8 and 16 years of ages (Figure 2C, Figure S2D).

\subsection{2 | Sensitization}

CXCL10 levels in cord blood were significantly lower than in nonsensitized children at 1 and 16 years of ages (Figure S3A, Figure 3A). Similarly, CXCL11 levels at 1 year and at birth were significantly elevated in non-sensitized children at 1 and 16 years of age, respectively (Figure S3B and C). Children who were sensitized at 1 , 8,11 and 16 years of ages consistently displayed significantly elevated concentrations of CCL18 at 1 and/or 8 years compared to
(A)

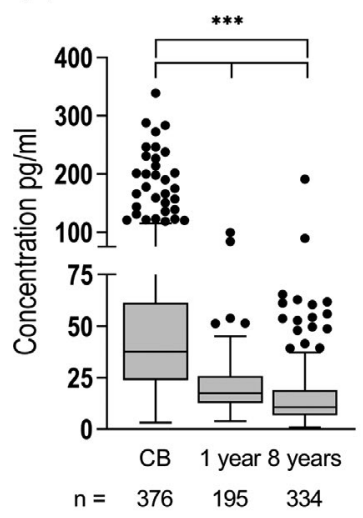

(D)
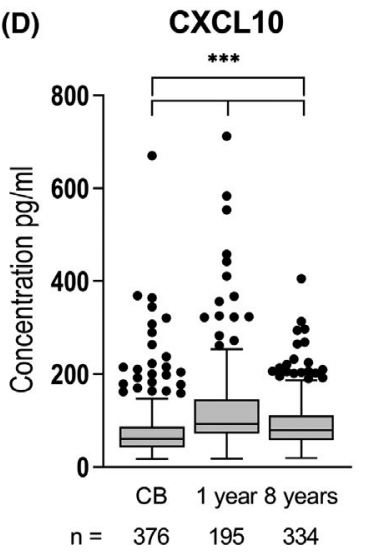

(B)

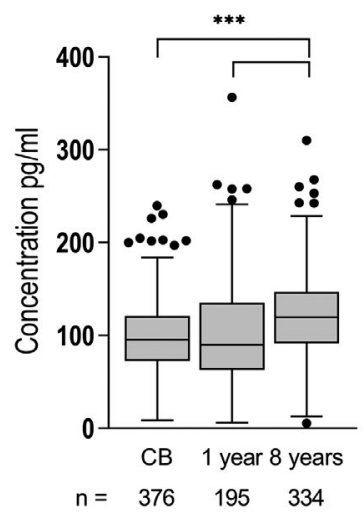

(E)

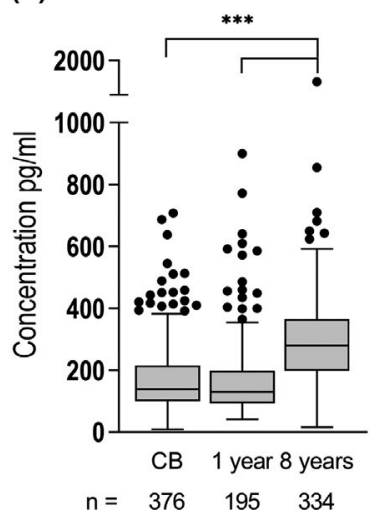

(C)

CCL18

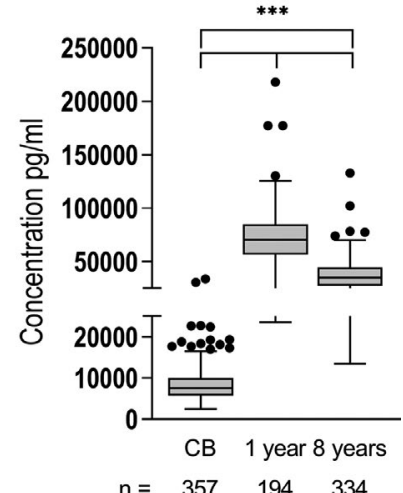

$n=357 \quad 194 \quad 334$
FIGURE 1 Circulating levels of chemokines from children in the MAAS cohort. Plasma concentrations of (A) CCL17, (B) CCL22, (C) CCL18, (D) CXCL10 and (E) CXCL11 were measured by the means of Luminex and ELISA methodology at birth (in cord blood), age 1 and age 8 . The data are displayed as medians with interquartile ranges. Statistical differences were ascertained using a Kruskal-Wallis test with a Dunn's post hoc test for multiple comparisons. ${ }^{* * *} p<.001$. CB, cord blood 

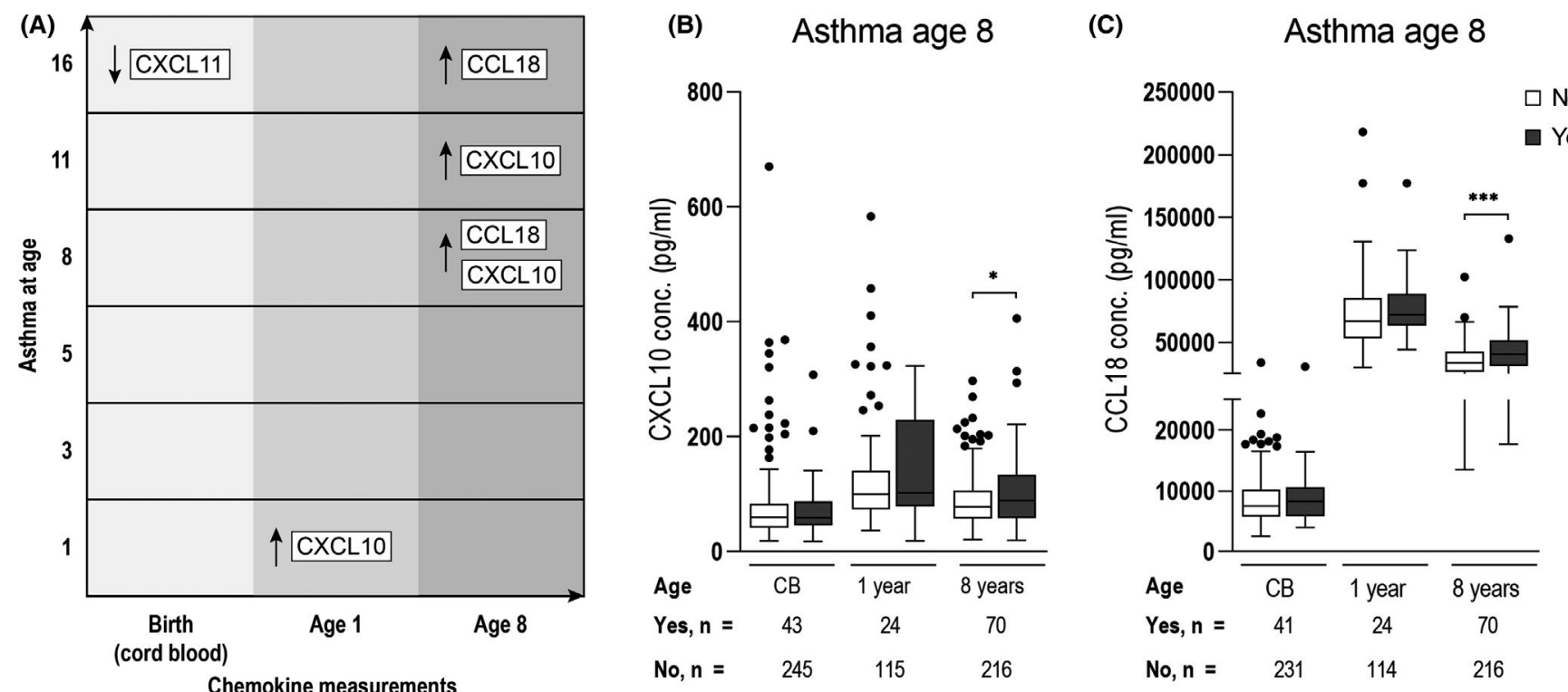

FIGURE 2 Associations of the allergy-related chemokines with asthma development. An overview of the significant comparisons of the chemokine levels at birth, ages 1 and 8 in relation to the development of asthma at 1-16 years of ages, is provided in (A). Increased or decreased levels are marked with an arrow in the corresponding direction. Plasma concentrations of the Th1-associated chemokine CXCL10 are displayed in relation to development of asthma at age 8 in (B). Circulating levels of the Th2/Treg-associated chemokine CCL18 in relation to asthma development at age 8 in (C). Asthma was defined as fulfilling at least two out of three criteria at the investigated time point: current wheeze, current use of asthma medication or physician-diagnosed asthma. The data are presented as medians with interquartile ranges. Mann-Whitney $U$ tests were performed to survey statistical significance. ${ }^{*} p<.05,{ }^{* * *} p<.001$. CB, cord blood

non-sensitized children (Figure 3C, Figure S4A-C). Increased levels of the Th2-associated chemokine CCL17 at birth associated with sensitization at age 8 (Figure S4D).

\subsection{CXCL10, CXCL11 and CCL18 predict asthma and sensitization}

As some of the chemokines indeed associated with the development of asthma and sensitization later in childhood, we tested the ability of the chemokine levels to predict asthma (Figure 4A) and sensitization (Figure 4B) later in childhood and adolescence using binomial logistic regression models.

\subsection{1 | Asthma}

Having higher CXCL11 levels at birth constituted lower odds of being asthmatic at age $16(\mathrm{OR}=0.4,95 \% \mathrm{Cl} 0.2-0.8, p=.012)$. Furthermore, higher CCL18 levels at 8 years of age markedly increased the odds of being asthmatic at $8(\mathrm{OR}=3.8,95 \% \mathrm{Cl} 1.5-9.8$, $p=.006)$ and 16 years (OR $=5.3,95 \% \mathrm{Cl} 1.7-16.1, p=.003)$ of ages

\subsection{2 | Sensitization}

Presenting with elevated CCL18 levels at age 8 posed a three times or higher odds ratio for developing sensitization at 8 (OR $=3.3,95 \%$
Cl $1.5-7.1, p=.002), 11$ (OR = 3.0, 95\% Cl 1.3-6.7, $p=.008)$ and 16 years $(\mathrm{OR}=4.2,95 \% \mathrm{Cl} 1.7-10.5, p=.002)$ of ages. In contrast, having high levels of CXCL10 at age 8 seemed protective against becoming sensitized at age $11(\mathrm{OR}=0.4,95 \% \mathrm{Cl} 0.2-0.8, p=.012)$.

\subsection{Association between CCL18 levels and the development of asthma and sensitization}

Proceeding with the chemokines showing the strongest consistent associations cross-sectionally, we set up GEE models studying each chemokine at each age separately in relation to various intervals of asthma and sensitization development to study longitudinal relationships over time. These models take into account correlations within individuals, and as the data are not independent over time, this provides an advantage of running both the GEE and logistic regression models. Consistent longitudinal patterns were only revealed for circulating levels of CCL18 at ages 1 and 8 (Figure 5).

\subsection{1 | Asthma}

In a longitudinal model including information on asthma from four time points (ages 5, 8, 11 and 16), we observed a significant association between increasing CCL18 levels at 1 year of age and the higher risk of asthma from early school age to adolescence $(\mathrm{OR}=2.9,95 \% \mathrm{Cl}$ 1.1-7.6, $p=.028$ ). Similarly, higher CCL18 levels at 1 (OR $=3.5,95 \%$ $\mathrm{Cl} 1.3-9.8, p=.01)$ and 8 years $(\mathrm{OR}=3.0,95 \% \mathrm{Cl} 1.6-5.9, p=.001)$ 


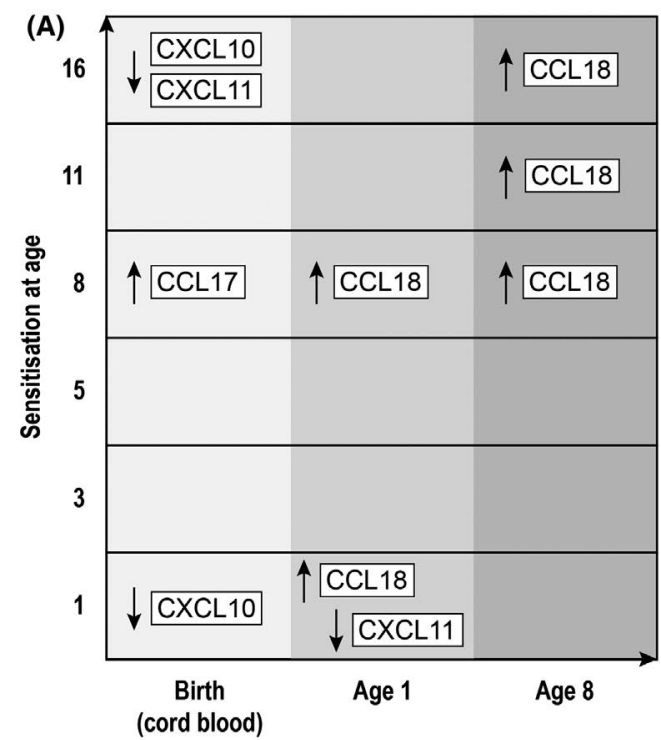

Chemokine measurements
(B) Sensitisation age 16

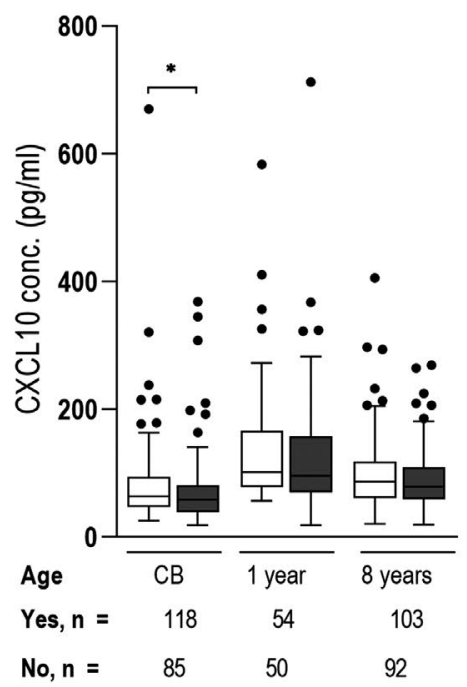

(C) Sensitisation age 16

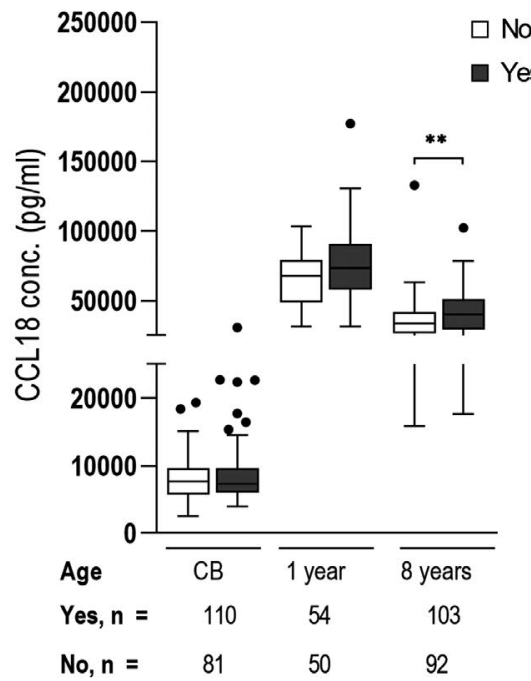

FIGURE 3 Associations of the allergy-related chemokines with allergic sensitization. An overview of the significant comparisons of the chemokine levels at birth, ages 1 and 8 in relation to development of sensitization at 1-16 years of ages, is provided in (A). Increased or decreased levels are marked with an arrow in the corresponding direction. Plasma concentrations of the Th1-associated chemokine CXCL10 are displayed in relation to development of sensitization, at 16 years of age in (B). Circulating levels of the Th2/Treg-associated chemokine CCL18 in relation to sensitization at 16 years of age are illustrated in (C). Sensitization status was determined by means of skin prick testing. The data are displayed as medians with interquartile ranges. Mann-Whitney $U$ tests were performed. ${ }^{*} p<.05,{ }^{* *} p<.01$. CB, cord blood
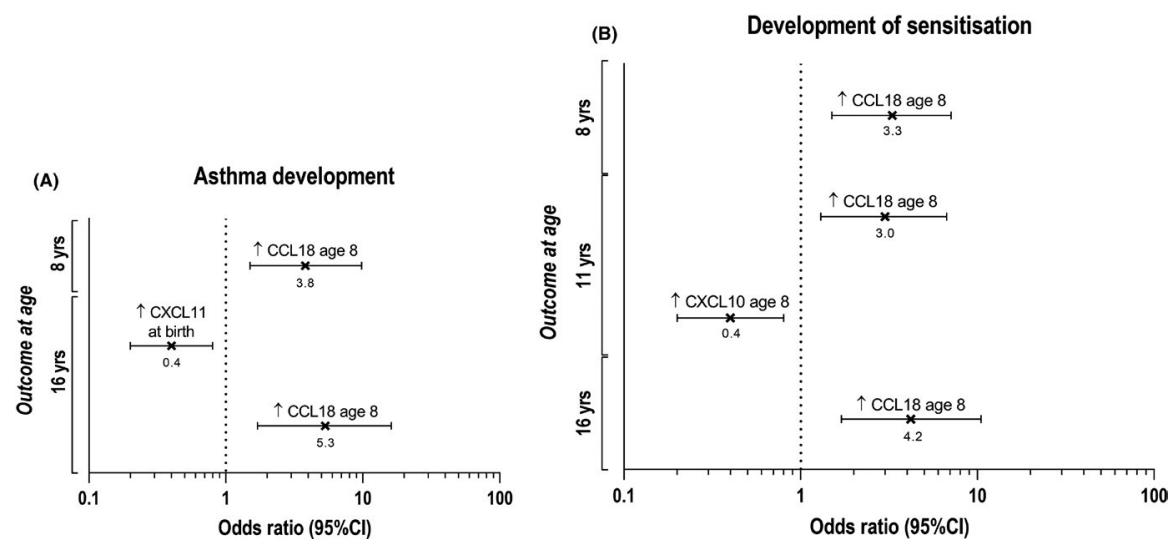

FIGURE 4 Forest plots of odds ratios from logistic regression models on predicting allergy development from circulating chemokine levels. Probabilities of developing asthma as depicted in (A) and sensitization as illustrated in (B) from cross-sectional adjusted logistic regression models including all chemokines and correction for the confounding factors such as sex, parental atopy and parental smoking. Odds ratios are denoted with crosses and the corresponding values below them, and $95 \%$ confidence intervals are illustrated by the error bars. All displayed models have an adjusted $p$-value of $<.05$

of ages were associated with an increased risk of asthma between 8 and 16 years of ages.

\subsection{2 | Sensitization}

In a longitudinal model including information on SPTs from five time points (3-16 years of ages), the odds of becoming sensitized increased significantly with increased CCL18 concentrations at age $1(\mathrm{OR}=3.1,95 \% \mathrm{Cl} 1.2-8.3, p=.022)$. Similarly, the odds ratios for developing sensitization between the 8 and 16 years of ages increased significantly with increasing CCL18 levels at $1(\mathrm{OR}=3.5$, $95 \% \mathrm{Cl} 1.3-9.9, p=.018)$ and 8 years $(\mathrm{OR}=3.0,95 \% \mathrm{Cl} 1.5-5.9$, $p=.002)$ of ages.

\subsection{Association of CCL18 levels with clusters of allergic diseases}

As we previously have derived clusters of allergic sensitization, ${ }^{14,15}$ allergic diseases ${ }^{16}$ and asthma exacerbations ${ }^{17}$ from children in the MAAS cohort using machine learning, we sought to study the 


\section{Longitudinal GEE model}

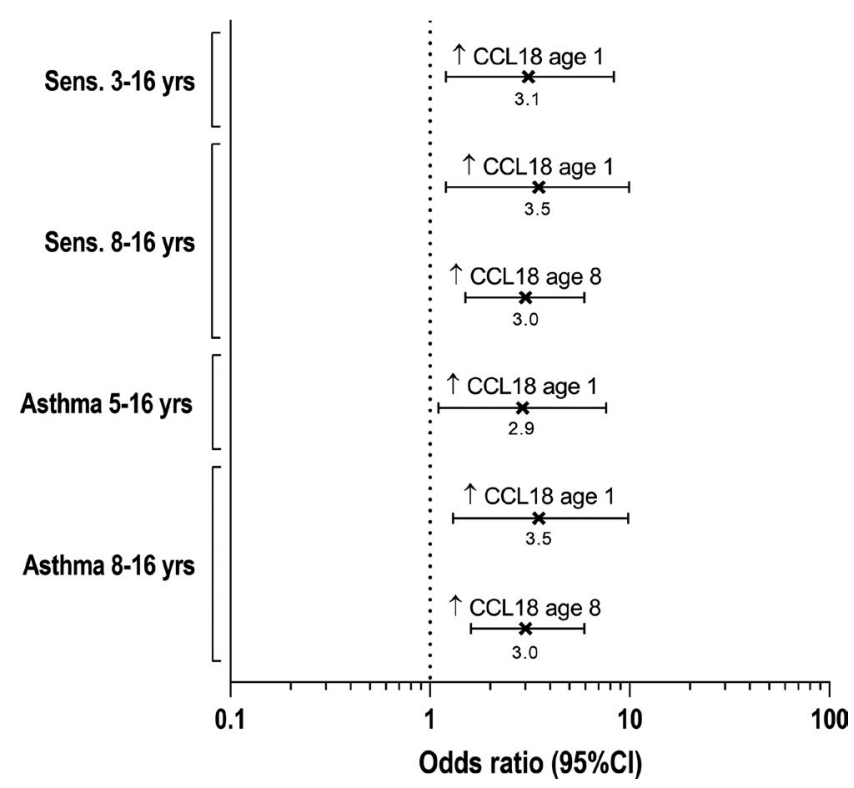

FIGURE 5 Forest plot of odds ratios from generalized estimation equation (GEE) models predicting longitudinal allergy development from the measured chemokines. In the GEE-models, the predictive ability of the chemokine CCL18 on longitudinal development of asthma at $5-16$ years and 8-16 years of ages, as well as sensitization at 3-16 years and 8-16 years of age, was examined. Odds ratios are denoted with crosses and the corresponding values below them, and $95 \%$ confidence intervals are illustrated by the error bars. All displayed models revealed an adjusted $p$-value of $<.05$

relationship between the chemokine levels and these putative endotypes of allergic diseases.

Children belonging to the multiple early sensitization cluster $^{14}$ had significantly higher levels of CCL18 at age 8 compared to non-atopic subjects (Figure 6A). In relation to CRD sensitization patterns, ${ }^{15}$ circulating levels of CCL18 at age 8 were elevated in individuals sensitized towards multiple allergens as compared to children with predominant grass and tree sensitization, and as a trend compared to children who were sensitized to a lesser degree (Figure 6B).

There was a trend towards higher levels of CCL18 at age 8 both in the eczema only and the atopic march clusters compared to the cluster consisting of healthy children ${ }^{16}$ (Figure 6C).

Associating chemokine levels with the developmental pattern of asthma exacerbations in the first 8 years of life, children presenting with exacerbations ${ }^{17}$ showed significantly higher levels of CCL18 at age 8 compared to non-wheezers (Figure 6D).

None of the other chemokines revealed any differences between the investigated clusters.

\section{4 | DISCUSSION}

In this study, we show that the levels of the chemokines CCL18, CXCL10 and CXCL11 in early life and childhood may predict outcomes of allergic disease later in childhood and adolescence. The main finding was that the dually Th2/Treg-regulated chemokine CCL18 predicted the development of both asthma and sensitization, with consistent effects over time. This corroborates our previous findings of elevated CCL18 levels in infancy of children developing eczema and recurrent wheeze. ${ }^{8}$ Being constitutively expressed in the lung and lymphoid tissues during homeostatic conditions by induction of IL-4, IL-13 and IL-10, CCL18 exhibits both chemotactic and immunoregulatory properties. ${ }^{12}$ It promotes tolerogenic differentiation of dendritic cells, which in turn may polarize $T$ cells into Tregs, and may polarize memory $\mathrm{T}$ cells into FoxP3+ $\mathrm{T}$ cells in vitro. In allergic subjects, however, the tolerogenic effects of CCL18 are seemingly abrogated, despite being upregulated in allergic conditions such as atopic dermatitis $8,18,19$ and asthma. ${ }^{12}$ Described being due to less efficient binding of the protein on immune cells, this possibly could partly explain the loss of tolerance in allergic individuals. Furthermore, CCL18 induces production of collagen in both the skin and the lung, implying a role in remodelling of the airways typically seen in asthmatic subjects. ${ }^{12}$ As alveolar macrophages are the main producers of CCL18 in the lung, where its expression is constitutive, it is tempting to speculate that these levels are augmented in asthmatic individuals owing to dysregulation of these cells. However, as our measurements were performed in plasma samples, and CCL18 may originate from one of many bodily sources, we cannot draw conclusions on tissue-specific effects of the observed elevation without performing functional studies. Furthermore, whether heightened CCL18 responses in asthmatic and sensitized children constitute causative mechanisms of allergy induction, or compensatory immune dampening responses, remains to be elucidated. We further examined chemokine expression within allergy clusters previously derived from our cohort. ${ }^{14-17}$ Indeed, CCL18 levels at age 8 were higher in the multiple early allergic sensitization cluster. Moreover, children with asthma exacerbations had higher levels of CCL18 at age 8 compared to children without wheeze. Taken together, this suggests that increased CCL18 levels later in childhood may reflect allergic disease severity, although further studies should elaborate on this matter.

Interesting findings also appeared for the Th1-associated chemokines CXCL10 and CXCL11. These chemokines are induced by IFN $-\gamma^{20}$ and are mainly expressed by the thymus, peripheral blood leukocytes, endothelial and epithelial cells. ${ }^{21,22}$ Elevated levels of CXCL10 in infancy and childhood associated with present and future development of asthma, in line with results from children with wheezing at age 3 , who subsequently developed asthma at age $6 .{ }^{11}$ Additionally, CXCL10 levels are increased in viral-induced asthma, ${ }^{23,24}$ suggesting that viral infections may induce Th1chemokine responses in asthmatic individuals. On the contrary, low cord blood CXCL10 levels associated with sensitization in infancy and adolescence. Similarly, decreased CXCL11 levels in early life associated with later development of sensitization. This corroborates findings from our previous studies, where SPT-positive children had lower levels of CXCL11 at birth and 24 months. ${ }^{8}$ As sensitization is a Th2-driven process, and Th1 responses were 

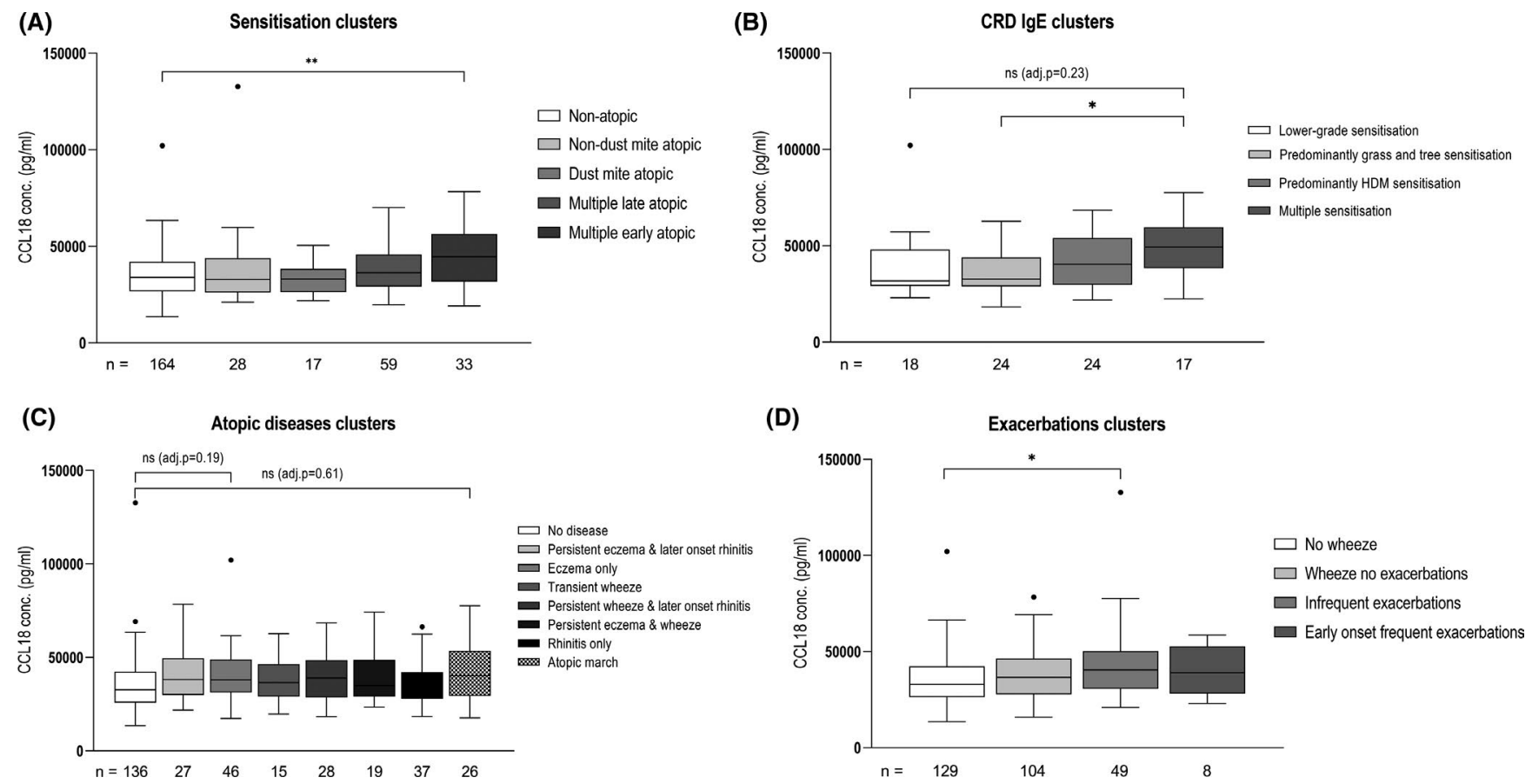

FIGURE 6 Associations of CCL18 levels at age 8 with previously machine learning-derived clusters of allergy outcomes from the MAAS cohort. Panel (A) displays sensitization clusters, (B) CRD IgE clusters, (C) atopic diseases clusters, and (D) exacerbations clusters. The data are presented as medians with interquartile ranges. A Kruskal-Wallis test with Dunn's post hoc test for multiple comparisons was performed. ${ }^{*} p<.05,{ }^{* *} p<.01$. CRD, component resolved diagnostics

lessened in sensitized children, diminished neonatal Th1 responses seemingly pave way for the development of sensitization in these children. Additionally, reduced cord blood CXCL11 levels associated with asthma at age 16 and translated into a predicted lower risk with high CXCL11 levels at birth, supporting previous results where children with the highest quartile CXCL11 levels at birth did not become sensitized throughout the first 2 years of life. ${ }^{8}$ No long-term effects of CXCL10 and CXCL11 on allergy development could be demonstrated in this study. Possibly, the function of Th1 cells, and their expression of IFN- $\gamma$, may become attenuated due to immunoregulatory effects of the highly expressed CCL18 on Tregs, although the findings may also constitute altered patterns of expression in allergic conditions. Collectively, this indicates that although these chemokines are induced by the same cytokine, downstream effects seem to be differentially regulated both in terms of allergy outcome and how levels reflect temporal development of disease.

There are both limitations and strengths to the present study. We evaluated circulating chemokine levels but did not have the opportunity to evaluate functional aspects of the same mediators in different tissues. This would have added mechanistic insights into the findings presented here. Also, the generalizability of these data may be limited, as children in the cohort originate from the Greater Manchester region, with rather homogenous populations. There were differences in sample size in the different age groups, originating from losses to follow-up over time, which may have limited the power of our analyses. However, as few studies have surveyed circulating chemokines at this magnitude, we believe that the substantial sample size still is a strength of this study. Furthermore, the apparent difference in parental atopy and asthma between included and excluded individuals is most probably by chance, as all children with available plasma samples were included for chemokine analysis. This was additionally taken into account by including these variables as co-variates in our models. The consistency of the methodologies used compared to previous studies provides another advantage. Moreover, by performing both cross-sectional logistic regression and longitudinal GEE models we have taken into account different temporal perspectives throughout childhood, which is a strength of this study.

To our knowledge, this is the first study to show that childhood circulating CCL18 levels may affect allergy-related outcomes longitudinally until adolescence. Further studies would, however, need to confirm the importance of CCL18, as well as CXCL10 and CXCL11, in the prediction of long-term development of allergic manifestations in a clinical setting. Possibly, circulating chemokine levels early in life could be used as biomarkers for the prediction of allergic disease and thereby contribute to timely preventive interventions and/or treatments.

In conclusion, we have shown that the elevated levels of CCL18 throughout childhood precede the development of asthma and sensitization, findings that remained solid longitudinally. The Th1associated chemokines CXCL10 and CXCL11 also predicted development of sensitization and asthma, with differential regulation at different time points in life. This motivates further investigations of chemokines as biomarkers for allergy development, with putative clinical utility in the prediction of allergic outcomes. 


\section{ACKNOWLEDGMENTS}

We would like to thank Anne-Marie Fornander for her outstanding technical assistance on the chemokine analyses, and Carolina Gunhardsson for her efforts with the CCL18 ELISA.

\section{CONFLICT OF INTEREST}

AS has received grants from MRC and Manchester Biomedical Research centre during conduct of the study. CSM reports lecture fees from GSK, Novartis, Astra Zeneca and Thermo Fisher Scientific. $A C$ has received consultancy and/or speaker fees from Novartis, Thermo Fisher Scientific, Philips, Sanofi and Stallergenes Greer. JH, $\mathrm{SH}$ and $\mathrm{MCJ}$ report no conflicts of interest.

\section{AUTHOR CONTRIBUTION}

Johanna Huoman: Conceptualization (supporting); Data curation (equal); Formal analysis (lead); Funding acquisition (supporting); Investigation (lead); Methodology (lead); Visualization (lead); Writing-original draft (lead); Writing-review \& editing (lead). Sadia Haider: Data curation (equal); Formal analysis (equal); Investigation (equal); Software (equal); Writing-original draft (supporting); Writingreview \& editing (supporting). Angela Simpson: Project administration (equal); Resources (equal); Writing-original draft (supporting); Writing-review \& editing (supporting). Clare Suzanne Murray: Project administration (equal); Resources (equal); Writing-original draft (supporting); Writing-review \& editing (supporting). Adnan Custovic: Conceptualization (equal); Funding acquisition (equal); Investigation (equal); Project administration (equal); Resources (equal); Supervision (equal); Writing-original draft (supporting); Writing-review \& editing (supporting). Maria Christina Jenmalm: Conceptualization (lead); Data curation (equal); Funding acquisition (equal); Investigation (equal); Methodology (equal); Supervision (lead); Writing-original draft (equal); Writing-review \& editing (equal).

\section{PEER REVIEW}

The peer review history for this article is available at https://publo ns.com/publon/10.1111/pai.13574.

\section{DATA AVAILABILITY STATEMENT}

The data that support the findings of this study are available from the corresponding author upon reasonable request.

\section{ORCID}

Johanna Huoman (D) https://orcid.org/0000-0003-2509-2418

Adnan Custovic (D) https://orcid.org/0000-0001-5218-7071

Maria C. Jenmalm (D) https://orcid.org/0000-0002-2117-5366

\section{REFERENCES}

1. Thomsen SF. Epidemiology and natural history of atopic diseases. Eur Clin Respir J. 2015;2(1):24642.

2. Akar-Ghibril N, Casale T, Custovic A, Phipatanakul W. Allergic endotypes and phenotypes of asthma. J Allergy Clin Immunol Pract. 2020;8(2):429-440.
3. Oksel C, Haider S, Fontanella S, Frainay C, Custovic A. Classification of pediatric asthma: from phenotype discovery to clinical practice. Front Pediatr. 2018;6:258.

4. Howard R, Rattray M, Prosperi M, Custovic A. Distinguishing asthma phenotypes using machine learning approaches. Curr Allergy Asthma Rep. 2015;15(7):38.

5. Pease J, Williams T. Chemokines and their receptors in allergic disease. J Allergy Clin Immunol. 2006;118(2):305-318.

6. Sandberg M, Frykman A, Ernerudh J, et al. Cord blood cytokines and chemokines and development of allergic disease. Pediatr Allergy Immunol. 2009;20(6):519-527.

7. Abelius MS, Ernerudh J, Berg G, Matthiesen L, Nilsson LJ, Jenmalm MC. High cord blood levels of the T-helper 2-associated chemokines CCL17 and CCL22 precede allergy development during the first 6 years of life. Pediatr Res. 2011;70(5):495-500.

8. Abrahamsson TR, Sandberg Abelius M, Forsberg A, Björkstén B, Jenmalm MC. A Th1/Th2-associated chemokine imbalance during infancy in children developing eczema, wheeze and sensitization. Clin Exp Allergy. 2011;41(12):1729-1739.

9. Southworth T, Pattwell C, Khan N, et al. Increased type 2 inflammation post rhinovirus infection in patients with moderate asthma. Cytokine. 2020;125:154857.

10. Ghebre MA, Pang PH, Desai D, et al. Severe exacerbations in moderate-to-severe asthmatics are associated with increased proinflammatory and type 1 mediators in sputum and serum. BMC Pulm Med. 2019;19(1):144.

11. Reubsaet LL, Meerding J, de Jager W, et al. Plasma chemokines in early wheezers predict the development of allergic asthma. Am J Respir Crit Care Med. 2013;188(8):1039-1040.

12. Chenivesse C, Tsicopoulos A. CCL18 - beyond chemotaxis. Cytokine. 2018;109:52-56. https://doi.org/10.1016/j.cyto.2018.01.023

13. Custovic A, Simpson BM, Murray CS, Lowe L, Woodcock A. The national asthma campaign manchester asthma and allergy study. Pediatr Allergy Immunol. 2002;13(s15):32-37.

14. Simpson A, Tan VYF, Winn J, et al. Beyond atopy. Am J Respir Crit Care Med. 2010;181(11):1200-1206.

15. Fontanella S, Frainay C, Murray CS, Simpson A, Custovic A. Machine learning to identify pairwise interactions between specific IgE antibodies and their association with asthma: a cross-sectional analysis within a population-based birth cohort. PLoS Medicine. 2018;15(11):e1002691.

16. Belgrave DC, Granell R, Simpson A, et al. Developmental profiles of eczema, wheeze, and rhinitis: two population-based birth cohort studies. PLoS Medicine. 2014;11(10):e1001748.

17. Deliu M, Fontanella S, Haider S, et al. Longitudinal trajectories of severe wheeze exacerbations from infancy to school age and their association with early-life risk factors and late asthma outcomes. Clin Exp Allergy. 2020;50(3):315-324.

18. Hon K, Ching GK, Ng P, Leung T. Exploring CCL18, eczema severity and atopy: PARC and eczema. Pediatr Allergy Immunol. 2011;22(7):704-707.

19. Günther C, Bello-Fernandez C, Kopp T, et al. CCL18 is expressed in atopic dermatitis and mediates skin homing of human memory $T$ cells. J Immunol. 2005;174(3):1723-1728.

20. Groom JR, Luster AD. CXCR3 ligands: redundant, collaborative and antagonistic functions. Immunol Cell Biol. 2011;89(2):207-215.

21. Romagnani P, Annunziato F, Lazzeri E, et al. Interferon-inducible protein 10, monokine induced by interferon gamma, and interferon-inducible T-cell alpha chemoattractant are produced by thymic epithelial cells and attract T-cell receptor (TCR) $\alpha \beta+C D 8+$ single-positive T cells, TCR $\gamma \delta+$ T cells, and natural killer-type cells in human thymus. Blood. 2001;97(3):601-607.

22. Cole KE, Strick CA, Paradis TJ, et al. Interferon-inducible T cell alpha chemoattractant (I-TAC): a novel Non-ELR CXC chemokine 
with potent activity on activated T Cells through selective high affinity binding to CXCR3. J Exp Med. 1998;187(12):2009-2021.

23. Wark PAB, Bucchieri F, Johnston SL, et al. IFN- $\gamma$-induced protein 10 is a novel biomarker of rhinovirus-induced asthma exacerbations. J Allergy Clin Immunol. 2007;120(3):586-593.

24. Moskwa S, Piotrowski W, Marczak J, et al. Innate immune response to viral infections in primary bronchial epithelial cells is modified by the atopic status of asthmatic patients. Allergy Asthma Immunol Res. 2018;10(2):144.

\section{SUPPORTING INFORMATION}

Additional supporting information may be found online in the Supporting Information section.
How to cite this article: Huoman J, Haider S, Simpson A, Murray CS, Custovic A, Jenmalm MC. Childhood CCL18, CXCL10 and CXCL11 levels differentially relate to and predict allergy development. Pediatr Allergy Immunol. 2021;32:1824-1832. https://doi.org/10.1111/pai.13574 